\title{
Evaluación anual del fitoplancton y su respuesta a la calidad de agua en el lago de Amatitlán, Guatemala
}

\author{
Annual evaluation of phytoplankton and its answer to water quality \\ in Amatitlán lake, Guatemala \\ Evelyn Rodas-Pernillo ${ }^{1 *}$, Camila A. Vasquez-Moscoso ${ }^{2}$ \\ 'Doctorado en Ciencias de la Investigación, Universidad Mariano Gálvez, Guatemala y \\ ${ }^{2}$ Departamento de Biología y Química, Facultad de Ciencias Básicas e Ingenierías, Universidad de los Llanos, Colombia
}

*Autor al que se dirige la correspondencia: rodaspernillo@gmail.com

Recibido: 29 de enero 2019 / Revisión: 22 de octubre 2019 / Aceptado: 09 de junio 2020

\section{Resumen}

Se realizó un estudio sobre la composición y abundancia del fitoplancton en el lago de Amatitlán, y el efecto de la calidad de agua sobre su biodiversidad. Para ello, se colectaron muestras de agua en cuatro puntos específicos del lago, en la superficie del agua y a profundidades de 5, 10 y $20 \mathrm{~m}$, de manera mensual durante el 2017. Se midieron parámetros fisicoquímicos in situ como temperatura y $\mathrm{pH}$. Igualmente, se identificaron y contabilizaron cianobacterias y microalgas. El índice de estado trófico (IETP) catalogó al lago como eutrófico e hipertrófico $($ IETP $=63.80-88.18)$. Se reportan 34 géneros de fitoplancton distribuidos en 30 familias, 17 órdenes y 10 clases. Los indicadores biológicos, tales como, floraciones algales de Microcystis (38.41\%), baja diversidad de diatomeas (Nitzschia, Aulacoseira y Cyclotella), presencia de microalgas Nitzschia y Scenedesmus resistentes a procesos de eutrofización, y alta concentración de coliformes fecales, de hasta 24,000 NMP/100 ml, evidenciaron la baja calidad de agua que se presenta en el lago de Amatitlán. En época seca se encontró más diversidad de microalgas debido a la mayor incidencia de radiación solar, el poco recambio de agua y la acumulación de materia orgánica. Aunque esto varía con los cambios en la concentración de nitrógeno total (NT) y fosforo total (PT), que potencian la proliferación de cianobacterias tóxicas. La biodiversidad del lago fue baja debido al estado hipereutrófico en que se encuentra. Se recomienda poner en funcionamiento plantas de tratamiento de aguas residuales para evitar que esta problemática continúe.

Palabras claves: Microalgas, aguas residuales, floraciones algales, Microcystis, eutrofización

Abstract

A study was carried out on the composition and abundance of phytoplankton in Amatitlán lake, and the effect of water quality on its biodiversity. For this, water samples were collected at four specific points in the lake, on the water surface and at depths of 5, 10 and $20 \mathrm{~m}$, in a monthly way during 2017. Physicochemical parameters were measured in situ such as temperature and $\mathrm{pH}$. Likewise, cyanobacteria and microalgae were identified and accounted. The trophic state index (IETP) cataloged the lake as eutrophic and hypertrophic (IETP=63.80-88.18). 34 genera of phytoplankton distributed in 30 families, 17 orders and 10 classes are reported. Biological indicators, such as Microcystis algal blooms (38.41\%), low diatom diversity (Nitzschia, Aulacoseira and Cyclotella), presence of Nitzschia and Scenedesmus microalgae resistant to eutrophication processes, and high concentration of fecal coliforms, up to 24,000 NMP/100 ml, evidenced the low quality of water that occurs in lake Amatitlán. In the dry season, more microalgae diversity was found due to the higher incidence of solar radiation, little water change and the accumulation of organic matter. Even though this varies with changes in the concentration of total nitrogen (NT) and total phosphorus (PT), which enhance the proliferation of toxic cyanobacteria. The lake's biodiversity was low due to its hypereutrophic state. We recommend to put this wastewater treatment plants into operation to prevent this problem to continue.

Keywords: Microalgae, wastewater, algal blooms, Microcystis, eutrophication 


\section{Introducción}

El fitoplancton es un conjunto heterogéneo de microorganismos fotosintéticos, denominado genéricamente como microalgas, las cuales pueden tener formas unicelulares, coloniales o filamentosas (Gómez, 2007). Su importancia ecológica radica en que son organismos autótrofos, productores primarios y fuente de alimento, rico en proteínas, vitaminas y minerales, para cladóceros, copépodos, larvas de algunos insectos y peces (Rodrigues et al., 2003).

La cuenca del lago de Amatitlán se considera como una de las más contaminadas de Centroamérica, aun así, se permite la pesca y la recreación. Estos niveles de contaminación se han incrementado sustancialmente desde 1950 debido a la deforestación, la agricultura intensiva y las aguas residuales industriales y domésticas que descargan por la corriente del río Villalobos, lo que ha llevado a la eutrofización del lago (Calderón, 2010). Adicionalmente, su efluente, el río Michatoya, es utilizado para producción de energía hidroeléctrica, generando diversos beneficios para la sociedad (Michels et al., 2014).

La eutrofización ocurre cuando hay exceso de concentración de nutrientes en un sistema hídrico, fundamentalmente nitrógeno y fósforo, lo que genera cambios en los factores fisicoquímicos del agua y a su vez en la fauna y flora que lo habitan (Murilloet al., 2010; Prosperi et al., 2005). Esto origina un crecimiento desmedido de algunas especies de fitoplancton, en especial de las cianobacterias, las cuales bajo ciertas condiciones ambientales (temperaturas elevadas, alta carga de nutrientes y $\mathrm{pH}$ alcalino) desarrollan floraciones (Reynolds, 1984), lo que hace que resulte un grupo competitivamente exitoso con relación a otros del fitoplancton (Paerl \& Huisman, 2009; Vera-Parra et al., 2011).

Las cianobacterias son microorganismos procariotas y los fotosintetizadores aeróbicos más antiguos del planeta, cuyas formas coloniales suelen formar agregados macroscópicos que resultan en floraciones, que deterioran la calidad del agua para consumo y fines recreativos (Lucena, 2008; Paerl \& Huisman, 2009). Afectan el desarrollo de la flora acuática porque impiden la llegada de luz a algunos sectores del lago, igualmente disminuyen la concentración de oxígeno disuelto necesario para peces e invertebrados. Además, hay especies de floraciones que producen cianotoxinas perjudiciales para la vida acuática y el ser humano, por ejemplo, Microcystis, una cianobacteria dominante en el lago de Amatitlán, se ha reportado como la responsable de grandes floraciones y productora de microcistinas, altamente tóxicas (Romero-Oliva et al., 2014; Falconer, 2005; Harke et al., 2016).

La intervención antrópica que ha sufrido el lago de Amatitlán ha modificado los parámetros fisicoquímicos del agua, al igual que las comunidades de fitoplancton, por lo que una bioevaluación de estos parámetros y su relación con las microalgas es relevante para determinar el estado del lago y establecer posibles soluciones (Gómez et al., 2001). La abundancia, composición y distribución del fitoplancton ha sido catalogado como indicador biológico de la calidad del agua (Plataroti \& Patiño, 2010), debido a que poseen un ciclo de vida corto y el recambio de especies es sensible a los cambios ambientales (Quiroz et al., 2004). Es por ello que en el lago de Amatitlán se han efectuado diversos estudios sobre la diversidad del fitoplancton y su potencial en la biotecnología (Calderón 2010; Díaz, 2011; Peckham $\&$ Dineen, 1953).

En el presente estudio se caracteriza el fitoplancton a diferentes profundidades de la columna de agua y su relación con algunas variables ambientales, con el fin de obtener las bases para la identificación de especies indicadoras de calidad de agua y proveer datos que permitan el estudio del potencial biotecnológico de las microalgas del lago de Amatitlán.

\section{Materiales y métodos}

\section{Área de Estudio}

El lago de Amatitlán (1428'34.6” N 90³5'16.0" O) se ubica en el municipio del mismo nombre a 26 $\mathrm{km}$ de la capital de Guatemala. Se encuentra a 1,186 m s.n.m. con una precipitación media anual sobre el lago de $1,220 \mathrm{~mm}$, la temperatura media anual es de $24.5^{\circ}$ $\mathrm{C}$, el número de horas de brillo solar es de $1.716 \mathrm{~h} / \mathrm{año}$ y la radiación solar de $18.6 \mathrm{MJ} / \mathrm{m}^{2} /$ día (Díaz, 2011). La cuenca del lago de Amatitlán es de origen vulcano-tectónico y es el cuarto lago con mayor extensión en Guatemala, con un área de $15.2 \mathrm{~km}^{2}$ (Gudiel, 2007).

Los muestreos de los parámetros fisicoquímicos para determinar la calidad del agua y el fitoplancton, se realizaron en cuatro puntos de muestreo (Figura 1): Este Centro (EC), Oeste Centro (OC), bahía Playa de Oro (BPO) y río Michatoya (RM), a profundidades de $0,5,10$ y $20 \mathrm{~m}$. Los sitios de muestreo del presente estudio fueron los puntos de monitoreo establecidos 


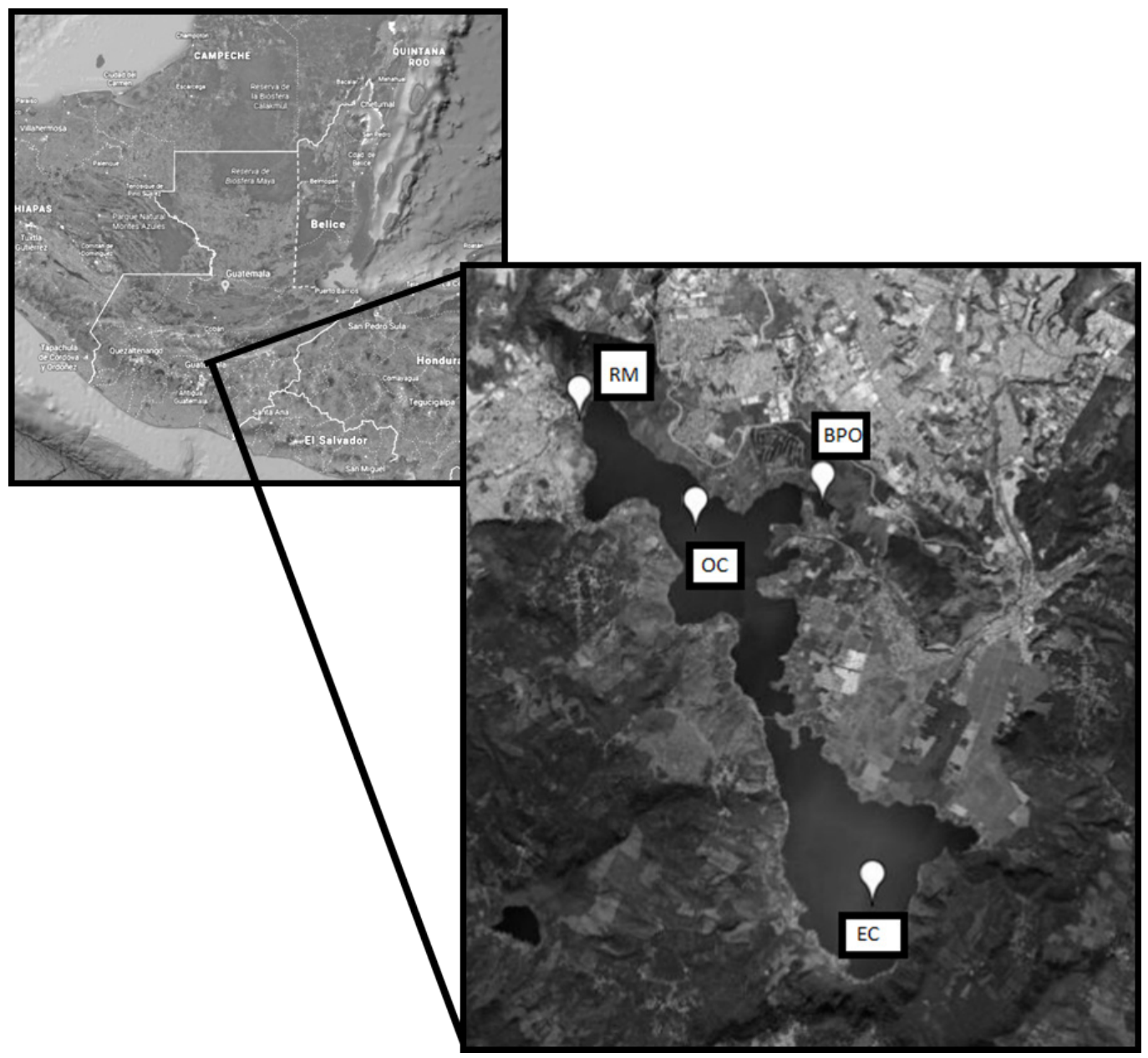

Figura 1. Localización de los sitios de muestro, dentro del Lago de Amatitlán (Guatemala). Este centro (EC): 14²5'52.5”

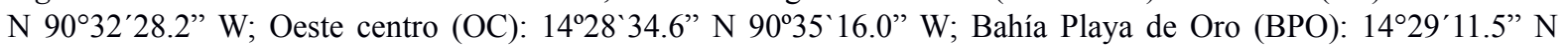
90³4'11.9” W; Río Michatoya (RM): 14²9’12.3” N 90³6’42.14” W.

previamente por la Autoridad para el Manejo Sustentable de la Cuenca y del Lago de Amatitlán (AMSA). El lago presenta dos subregiones divididas por una construcción denominada relleno para paso vehicular, en cada una de estas regiones se eligió su punto medio (EC y OC). La parte este del lago mide $7.12 \mathrm{Km}^{2}$ y la parte Oeste $8.23 \mathrm{Km}^{2}$ (Basterrechea, 1983). EC está ubicado en una parte del lago que tiene escasa comunicación con otros cuerpos hídricos, mientras que OC se encuentra entre el río Villalobos (afluente) y el río Michatoya (efluente), BPO se encuentra cercano a la desembocadura del río Villalobos y RM se ubica en el primer tramo del río Michatoya, cerca de la playa pública (Michels et al., 2014).

\section{Diseño de muestreo}

Los muestreos para medir los parámetros fisicoquímicos y determinar la calidad del agua, y los análisis cualitativos y cuantitativos para estudio de fitoplancton, fueron realizados mensualmente durante el 2017, en los cuatro puntos de muestreo que AMSA ha utilizado para monitorear la calidad de agua, EC, OC, BPO y RM (Figura 1). En los cuatro puntos se tomaron muestras de la superficie directamente. Adicionalmente, en EC y $\mathrm{OC}$ se muestreo a 10 y $20 \mathrm{~m}$ de profundidad y en BPO a $5 \mathrm{~m}$ con una botella Van Dorn de $5 \mathrm{~L}$. Este procedimiento se hizo por triplicado en cada punto de muestreo, llenando dos frascos plásticos de boca ancha 
con tapón de rosca de 1 L. Así mismo, se tomaron las muestras en frascos ámbar de $500 \mathrm{~mL}$ para el análisis de fitoplancton, y se adicionó lugol al 3\%. Seguidamente, todos los frascos fueron almacenados a $4{ }^{\circ} \mathrm{C}$ hasta su correspondiente análisis (Bellinger \& Sigee, 2015).

\section{Variables físicas y químicas}

Con el objetivo de considerar la variabilidad temporal de los parámetros fisicoquímicos y microbiológicos en el lago de Amatitlán, se realizaron análisis in situ y además se recolectaron muestras en los cuatro puntos de muestro para analizarlas en el Laboratorio de Control de la Calidad Ambiental de AMSA.

Los parámetros evaluados fueron: Temperatura, conductividad eléctrica y oxígeno disuelto con la sonda WTW Oxi 1970i; pH con el potenciómetro HQ 30d Marca HACH Flexi y la turbidez con el turbidímetro HACH 20100Q. Por otra parte, el nitrógeno total se midió siguiendo la metodología de Müller y Weideman (1955), fósforo total, ortofosfatos, sólidos totales suspendidos y disueltos, coliformes totales y coliformes fecales fueron analizados siguiendo los métodos estándar (Clesceri et al., 2005). La demanda química de oxígeno (Closed Reflux. 5220 D Colorimetric Method), tensoactivos (Spectroquant, prueba en cubetas tensoactivos aniónicos), clorofila $a$ (Henriques, M., Silva, A., \& Rocha, J. M.,2007), silicatos (Spectroquant, prueba de silicatos, ácido salicílico), y arsénico por medio de espectrofotometría de absorción atómica-horno de grafito (Clesceri, et al., 2005).

El Índice de Estado Trófico (IETP) se determinó a partir de la concentración de clorofila a y fósforo total, del agua más superficial, cuya relación con la transparencia se calculó previamente, siguiendo la metodología propuesta por Carlson (1977).

\section{Análisis cualitativo de fitoplancton}

Para identificar los géneros de fitoplancton presentes en la muestra se utilizó un microscopio invertido (Labomed TCM400) a un aumento total de 40X y $100 \mathrm{X}$, y se utilizaron las claves de Bellinger y Sigee (2015), Komárková y colaboradores (2016) y Cronberg y Annadotter (2006).

\section{Análisis cuantitativo de fitoplancton}

Se efectuó utilizando la cámara Sedgwick-Rafter y un microscopio invertido (Labomed TCM400) a un aumento total de 40X, contando un total de 100 unidades naturales (colonias, filamentos o células) por muestra, con el objetivo de llegar a un valor estadísticamente significativo (error del $\pm 20 \%$, con límites de confianza de $95 \%$ ). Se escogió este valor, ya que, para reducir dicho error a la mitad, habría que cuadriplicar los recursos para llevar a cabo esta cuantificación (Clesceri et al., 2005). La matriz para el análisis se expresó en abundancias relativas, es decir, el porcentaje de células/ $\mathrm{mL}$ de cada género reportado, por punto de muestreo.

\section{Análisis estadístico}

Calidad de agua: El análisis estadístico descriptivo se aplicó a 15 parámetros fisicoquímicos, uno microbiológico (coliformes fecales) y al IETP. Se calcularon los valores promedio, mínimo y máximo para estos parámetros, en cada punto de muestreo y profundidad.

Análisis cualitativo de fitoplancton: Para analizar la diversidad alfa entre sitios y meses de muestreo se calcularon índices de diversidad empleando el programa Past 3.15 (Hammer et al., 2001). Se calculó la diversidad de Shannon-Wiener, dominancia de Simpson y uniformidad de Pielou (Jost, 2006). Para esto se utilizó la matriz que discrimina la abundancia relativa por punto de muestreo (expresada en porcentaje de células/ $\mathrm{mL}$ ) de cada género identificado, y agrupando todos los meses y profundidades (Tabla 4). Los resultados obtenidos se transformaron a números de diversidad de Hill (1973).

Análisis cuantitativo de fitoplancton: Se realizó la prueba de Shapiro-Wilk, basada en la comparación de los cuartiles de la distribución normal, para determinar el tipo de distribución de los datos. Siguiendo estos resultados, se realizaron pruebas ANOVA para la riqueza e índices de diversidad, mientras que para la abundancia se desarrolló la prueba de Kruskal-Wallis, ya que su distribución no fue normal. Estas pruebas se hicieron con el software Statgraphicas Centurion XVI versión 16.1.03.

Efecto de la calidad del agua sobre la biodiversidad del fitoplancton: Se hizo un Análisis de Correspondencia Segmentado (DCA, por sus siglas en inglés) para establecer la técnica de ordenación que mejor se ajustaba a los datos obtenidos de abundancia del fitoplancton, en el software Canoco 4.5 (Leps \& Smilauer, 2003). Con base en este resultado se hizo un 
análisis de redundancia (RDA) para la ordenación de los géneros, con respecto a las variables ambientales de los sitios muestreados. Para este análisis se utilizaron los 34 géneros de microalgas encontradas durante el 2017 y 18 variables representativas.

\section{Resultados}

\section{Variables fisicoquímicas para determinar la calidad del agua}

El promedio anual de $\mathrm{pH}$ presentó valores entre 7.7 y 8.9 en los diferentes puntos de muestreo, y la temperatura de $22.2{ }^{\circ} \mathrm{C}$ a $25.9{ }^{\circ} \mathrm{C}$ (Tabla 1 y Tabla 3 ). La conductividad presentó los más altos promedios anuales en RM $(782.0 \mu \mathrm{S} / \mathrm{cm})$ y OC $(803.0 \mu \mathrm{S} / \mathrm{cm})$, a $0 \mathrm{~m}$ (Tabla 1). A profundidades de 5, 10 y $20 \mathrm{~m}$ los valores más altos se presentaron en EC $(730.0 \mu \mathrm{S} / \mathrm{cm})$ y OC (786.0 $\mu \mathrm{S} / \mathrm{cm}$ ) (Tabla 3). La concentración de oxígeno disuelto fue mayor en $\mathrm{EC}$, a $0 \mathrm{~m}$, con un a valor de 19.4 $\mathrm{mg} / \mathrm{L}$. Sin embargo, a 10 y $20 \mathrm{~m}$ en este mismo sitio ya se reportaron valores de $0 \mathrm{mg} / \mathrm{L}$.

La turbidez fue más alta en BPO a 0 m (450.0 NTU) y en OC a 10 y 20 m (24.0 NTU). Con respecto al nitrógeno total (NT) y fósforo total (PT) se reportaron los valores más altos en RM, con $260.0 \mathrm{mg} / \mathrm{L}$ y 18.2 $\mathrm{mg} / \mathrm{L}$ respectivamente. El IETP y los coliformes fecales solo se registraron en la superficie, el IETP llegó a los valores más altos en RM (88.2) y los menores en OC (63.8) (Tabla 1). Los coliformes fecales presentaron los valores más altos en $\mathrm{OC}(24,000 \mathrm{NMP} / 100 \mathrm{~mL})$ y los más bajos en EC (1.8 NMP/100 mL). Los demás valores de los parámetros fisicoquímicos reportados se muestran en las Tablas 1, 2 y 3.

\section{Análisis cualitativo de fitoplancton}

La clase Cyanophyceae (cianobacterias) fue la de mayor riqueza con 13 géneros, mientras que, a nivel de familia, Chlorellaceae fue la más representativa con tres géneros. Las demás familias estuvieron representadas por uno o dos géneros (Tabla 4). Tres puntos de muestreo dentro del lago de Amatitlán mostraron resultados similares en cuanto a riqueza $(p=.98), \mathrm{RM}$ demostró diferencias significativas de los demás puntos $(p=$ 8.85E $\mathrm{E}^{-5}$; Tabla 6). BPO, fue el de mayor riqueza, con 31 géneros; y RM el menor con 18. Así mismo, los meses con mayor número de géneros reportados fueron enero y febrero, mientras que, los de menor riqueza fueron abril, mayo y septiembre (Figura 2).

Los índices de Shannon-Weaver, Simpson y Pielou (Tabla 5) en EC y OC presentaron igual número de especies, no obstante, OC tuvo mayor valor en el índice de Shannon (N1). RM fue el sitio que mostró valores diferentes en los índices con respecto a los demás sitios, aunque ninguno con diferencia significativa $(p>.05)$. Los resultados obtenidos demuestran una baja diversidad en todos los puntos de muestreo (Tabla 6).

\section{Análisis cuantitativo de fitoplancton}

Se cuantificaron 34 géneros de microalgas durante el 2017, distribuidas en 30 familias, 17 órdenes, 10 clases, seis divisiones (phylum) y tres reinos. Se observa la mayor abundancia de fitoplancton en el mes de septiembre (30.15\%), pero no hay diferencias significativas según la prueba de Kruskal-Wallis para datos no paramétricos $(p=.285$; Tabla 6$)$. En septiembre se observa el mayor porcentaje de abundancia de fitoplancton en RM (25.11\%). Los meses de menor abundancia relativa fueron enero (2.84\%), febrero (1.07\%), marzo $(0.48 \%)$, abril (2.07\%) y mayo (4.59\%), (Figura 3$)$. Al analizar la abundancia de fitoplancton por profundidades, se observó que la superficie del lago presentó la mayor abundancia relativa en todos los puntos de muestreo (Figura 5), específicamente en el punto RM (32.90\%). BPO fue el único sitio donde se tomó muestra a $5 \mathrm{~m}$ de profundidad, en donde se observa menor abundancia (9.29\%) que en la superficie (12.53\%). En OC y EC se tomaron muestras a 10 y $20 \mathrm{~m}$. En OC se evidencia una disminución de la abundancia relativa de $13.97 \%$ a $3.69 \%$, conforme aumenta la profundidad; mientras que en EC se presentó la mayor abundancia de fitoplancton a $10 \mathrm{~m}$ de profundidad (7.46\%).

Con respecto a la abundancia relativa de los géneros, la cianobacteria Microcystis (Figura 4) y Pseudanabaena dominaron con 38.41 y $34.99 \%$ respectivamente, seguidas por Merismopedia con $10.74 \%$, mientras que, la abundancia menor $(<0.006 \%)$ la presentaron los géneros Cosmarium, Characium, Pandorina, Closterium, Oocystis y Snowella (Tabla 4). BPO, EC, y OC comparten la mayoría de las especies, aunque varían en abundancia, mostrando dominancia de Pseudanabaena en los tres sitios (35.16\% a 59.12\%). Por el contrario $\mathrm{RM}$, presentó menos especies y una predominancia de Microcystis con $69.08 \%$ (Tabla 4). 
Tabla 1

Parámetros fisicoquímicos de los puntos de muestreo en aguas superficiales (0-0.5m)

\begin{tabular}{|c|c|c|c|c|}
\hline & $\mathrm{EC}$ & $\mathrm{OC}$ & BPO & $\mathrm{RM}$ \\
\hline Parámetro & $\begin{array}{c}\text { Promedio } \\
\text { (Mínimo -Máximo) }\end{array}$ & $\begin{array}{c}\text { Promedio } \\
\text { (Mínimo -Máximo) }\end{array}$ & $\begin{array}{c}\text { Promedio } \\
\text { (Mínimo -Máximo) }\end{array}$ & $\begin{array}{c}\text { Promedio } \\
\text { (Mínimo -Máximo) }\end{array}$ \\
\hline $\mathrm{pH}$ & $8.9(7.5-9.5)$ & $8.5(7.4-9.4)$ & $8.4(7.8-9.2)$ & $8.4(7.2-9.4)$ \\
\hline $\mathrm{T}\left({ }^{\circ} \mathrm{C}\right)$ & $25.0(22.1-27.6)$ & $25.6(22.7-28.8)$ & $25.9(23.0-29.2)$ & $25.1(23-28.1)$ \\
\hline Cond. $(\mu \mathrm{S} / \mathrm{cm})$ & $597.8(566-632)$ & $723.4(652-803)$ & 658.5 (604-760) & $734(657-782)$ \\
\hline $\mathrm{O}_{2}(\mathrm{mg} / \mathrm{L})$ & $11.2(0.7-19.4)$ & $8.9(0.8-17.9)$ & $8.7(2.0-21.2)$ & $7.8(0.0-17.1)$ \\
\hline Turbidez (NTU) & $29.5(9.0-100)$ & $34.7(3-160)$ & $83.8(13-450)$ & $79.6(7-270)$ \\
\hline $\mathrm{DQO}\left(\mathrm{mgO}_{2} / \mathrm{L}\right)$ & $82.1(27-256)$ & $69.2(20-230)$ & $272.2(26-2046)$ & $445.8(31-3545)$ \\
\hline PT (mg/L) & $0.44(0.08-2.15)$ & $0.76(0.41-1.59)$ & $1.68(0.21-9.78)$ & $2.58(0.33-18.19)$ \\
\hline OrtP (mg/L) & $0.11(0.01-0.23)$ & $0.43(0.21-0.69)$ & $0.13(0.0-0.2102)$ & $0.44(0.1395-0.6869)$ \\
\hline NT (mg/L) & $4.01(0.66-25)$ & 3.97 (0.94-14.17) & $12.53(0.65-108.40)$ & $25.05(1-260.03)$ \\
\hline Sil (mg/L) & $26.45(18-32.30)$ & $17.56(2.40-30.40)$ & $68.70(54-86.7)$ & $15.81(0.95-29.4)$ \\
\hline $\mathrm{SST}(\mathrm{mg} / \mathrm{L})$ & $38.75(7-179)$ & $38.79(3-189.5)$ & $188.29(7-1546)$ & $401.21(7.5-2840)$ \\
\hline TDS (mg/L) & $309.75(281-372)$ & $362.58(326-414)$ & $333(301-382)$ & $362.50(328-394)$ \\
\hline \multirow[t]{2}{*}{ TenA (mg/L) } & $0.24(0.09-0.57)$ & $0.28(0.12-0.41)$ & $0.53(0.1-2.4)$ & $0.38(0.13-1.50)$ \\
\hline & 231.59 (21.36- & & & $444.90(10.64-$ \\
\hline Clor $(\mu \mathrm{g} / \mathrm{L})$ & 1378.84) & $122.84(4.01-369.51)$ & $445.58(39.5-3447.02)$ & 1948.05) \\
\hline IETP & $69.47(64.44-81.57)$ & $70.88(63.80-75.76)$ & $72.75(66.73-88.41)$ & $73.60(63.98-88.18)$ \\
\hline \multicolumn{5}{|l|}{ ColFec } \\
\hline (NMP/100 ml) & $13.60(1.80-33)$ & $6323.94(180-24000)$ & $191.11(1.80-1700)$ & $2032.92(45-9200)$ \\
\hline As (mg/L) & $0.02(0.01-0.03)$ & $0.03(0.02-0.44)$ & $0.03(0.02-0.03)$ & $0.03(0.02-0.04)$ \\
\hline
\end{tabular}

Nota. Abreviaturas de parámetros: As: arsénico; Clor: clorofila a; ColFec: coliformes fecales; Cond: conductividad; DQO: demanda química de oxigeno; IETP: índice de estado trófico; NT: nitrógeno total; $\mathrm{O}_{2}$ : oxígeno disuelto; OrtP: ortofosfatos; PT: fosforo total; Sil: Silicatos; SST: Solidos totales suspendidos; T: temperatura; TDS: solidos totales disueltos; TenA: tensoactivos. Abreviaturas para puntos de muestreo: Lago de Amatitlán este centro (EC), Lago de Amatitlán oeste centro (OC), bahía Playa de Oro (BPO) y río Michatoya (RM). 


\section{Efecto de la calidad del agua sobre la biodiver- sidad del fitoplancton}

El RDA para determinar la relación entre géneros y variables fisicoquímicas explicó en sus dos primeros ejes el $91.8 \%$ de la varianza acumulada (suma de valores propios: 0.763). El RDA expone a Microcystis como el género más distante del conglomerado, mostrando una relación directa con PT, DQO, NT y TSS, además de encontrarse mayoritariamente en la superficie del lago (P0). Los géneros Pseudanabaena (Pse),
Merismopedia (Mer), Aphanocapsa (Apha) y Synechocystis (Syn), todos de la clase Cyanophyceae, presentaron una relación inversa con el oxígeno disuelto y directa con las profundidades de 5,10 y $20 \mathrm{~m}$, es decir, fueron más abundantes que en la superficie. Con respecto a las demás especies, no se observa una diferencia entre las profundidades. Nitzschia mostró relación directa con el aumento de $\mathrm{pH}$, mientras que Rhodomonas, Spirulina y Closterium mostraron relación inversa con el mismo. Los demás parámetros fisicoquímicos no presentaron una ordenación explicativa (Figura 6).

Tabla 2

Parámetros fisicoquímicos de los puntos de muestreo a $10 \mathrm{~m}$ y $20 \mathrm{~m}$

\begin{tabular}{|c|c|c|c|c|}
\hline & $\mathrm{EC}(10 \mathrm{~m})$ & $\mathrm{EC}(20 \mathrm{~m})$ & $\mathrm{OC}(10 \mathrm{~m})$ & $\mathrm{OC}(20 \mathrm{~m})$ \\
\hline Parámetro & $\begin{array}{c}\text { Promedio } \\
\text { (Mínimo -Máximo) }\end{array}$ & $\begin{array}{c}\text { Promedio } \\
\text { (Mínimo -Máximo) }\end{array}$ & $\begin{array}{c}\text { Promedio } \\
\text { (Mínimo -Máximo) }\end{array}$ & $\begin{array}{c}\text { Promedio } \\
\text { (Mínimo-Máximo) }\end{array}$ \\
\hline $\mathrm{pH}$ & $8.6(7.7-9.1)$ & $8.1(7.7-8.5)$ & $8(7.4-8.6)$ & $7.7(7.5-8)$ \\
\hline $\mathrm{T}\left({ }^{\circ} \mathrm{C}\right)$ & $23.2(21-24.9)$ & $22.2(20.8-23.4)$ & $23.4(21.4-24.9)$ & $22.9(21.2-24.5)$ \\
\hline Cond. $(\mu \mathrm{S} / \mathrm{cm})$ & $609(561-730)$ & $601.3(283-669)$ & $715.9(667-773)$ & $722.1(669-786)$ \\
\hline $\mathrm{O}_{2}(\mathrm{mg} / \mathrm{L})$ & $2.6(0.0-7.6)$ & $0.7(0.0-2.5)$ & $0.7(0.05-3.4)$ & $0.3(0.1-0.6)$ \\
\hline Turbidez (NTU) & $9.3(4-16)$ & $8.5(5-13)$ & $13.9(4-24)$ & $15.7(9-24)$ \\
\hline $\mathrm{DQO}\left(\mathrm{mgO}_{2} / \mathrm{L}\right)$ & $38.5(27-48)$ & $38.7(26-55)$ & $40.6(18-51)$ & $38.4(20-52)$ \\
\hline PT (mg/L) & $0.21(0.09-0.30)$ & $0.32(0.23-0.39)$ & $0.58(0.12-0.85)$ & $0.73(0.49-0.9)$ \\
\hline OrtP (mg/L) & $0.15(0.03-0.24)$ & $0.27(0.18-0.36)$ & $0.54(0.32-0.73)$ & $0.63(0.33-0.79)$ \\
\hline NT (mg/L) & $1.10(0.45-2.38)$ & $1.92(0.78-3.04)$ & $2.90(1.45-4.70)$ & $3.28(1.55-4.80)$ \\
\hline Sil $(\mathrm{mg} / \mathrm{L})$ & $27.20(20.70-32.20)$ & $28.19(26.10-30.60)$ & $21.53(9.90-31.40)$ & $22.13(11.70-30.20)$ \\
\hline $\mathrm{SST}(\mathrm{mg} / \mathrm{L})$ & $6.46(3.00-9)$ & $5(1.50-10.50)$ & $9.54(2.50-18)$ & $7.42(1.50-15.50)$ \\
\hline TDS (mg/L) & $307.42(282-366)$ & $313.08(282-363)$ & $359.50(339.00-388.00)$ & $359.67(331-394)$ \\
\hline TenA (mg/L) & $0.06(0.19-0.46)$ & $0.13(0.07-0.24)$ & $0.27(0.15-0.40)$ & $0.28(0.12-0.44)$ \\
\hline
\end{tabular}

Nota. Abreviaturas de parámetros: As: arsénico; Clor: clorofila a; ColFec: coliformes fecales; Cond: conductividad; DQO: demanda química de oxigeno; IETP: índice de estado trófico; NT: nitrógeno total; O2: oxígeno disuelto; OrtP: ortofosfatos; PT: fosforo total; Sil: Silicatos; SST: Solidos totales suspendidos; T: temperatura; TDS: solidos totales disueltos; TenA: tensoactivos. Abreviaturas para puntos de muestreo: Lago de Amatitlán este centro (EC), Lago de Amatitlán oeste centro (OC), bahía Playa de Oro (BPO) y río Michatoya (RM). 
Tabla 3

Parámetros fisicoquímicos de Bahía Playa de Oro (BPO) a $5 \mathrm{~m}$

\begin{tabular}{|c|c|}
\hline & $\mathrm{BPO}$ \\
\hline Variable & Promedio (Mínimo -Máximo) \\
\hline $\mathrm{pH}$ & $8.1(7.7-8.4)$ \\
\hline $\mathrm{T}\left({ }^{\circ} \mathrm{C}\right)$ & $24.3(21.8-26.3)$ \\
\hline Cond. $(\mu \mathrm{S} / \mathrm{cm})$ & $656(592-769)$ \\
\hline $\mathrm{O}_{2}(\mathrm{mg} / \mathrm{L})$ & $1.5(0.1-4.4)$ \\
\hline Turbidez (NTU) & $23.3(7-56)$ \\
\hline $\mathrm{DQO}\left(\mathrm{mg} \mathrm{O}_{2} / \mathrm{L}\right)$ & $49.9(18-105)$ \\
\hline $\mathrm{PT}(\mathrm{mg} / \mathrm{L})$ & $0.33(0.15-0.71)$ \\
\hline OrtP (mg/L) & $0.18(0.04-0.37)$ \\
\hline NT $(\mathrm{mg} / \mathrm{L})$ & $1.68(0.72-4.93)$ \\
\hline Sil (mg/L) & $68.78(51.50-82.30)$ \\
\hline $\mathrm{SST}(\mathrm{mg} / \mathrm{L})$ & $17.04(9-48)$ \\
\hline $\mathrm{TDS}(\mathrm{mg} / \mathrm{L})$ & $329.50(304-382)$ \\
\hline TenA (mg/L) & $0.31(0.10-1.19)$ \\
\hline
\end{tabular}

Nota. As: arsénico; Clor: clorofila a; ColFec: coliformes fecales; Cond: conductividad; DQO: demanda química de oxigeno; IETP: índice de estado trófico; NT: nitrógeno total; O2: oxígeno disuelto; OrtP: ortofosfatos; PT: fosforo total; Sil: Silicatos; SST: Solidos totales suspendidos; T: temperatura; TDS: solidos totales disueltos; TenA: tensoactivos.

\section{Discusión}

En el lago de Amatitlán los valores de oxígeno disuelto indicaron un nivel de anoxia $(<2 \mathrm{mg} / \mathrm{L})$ en profundidades de 5, 10 y $20 \mathrm{~m}$, en todos los sitios muestreados. Esto puede estar sucediendo por el in- cremento en la producción de materia orgánica que es descompuesta por bacterias heterotróficas, las cuales a su vez aumentan la tasa de consumo de oxígeno. En estas condiciones se aumenta la liberación de fósforo de los sedimentos y los fosfatos $\left(\mathrm{PO}_{4}\right)$ se vuelven más inestables y disponibles para el crecimiento de fitoplancton. Así, los niveles de oxígeno pueden controlar la forma y disponibilidad de nutrientes esenciales para las microalgas. En situaciones en las que el nivel de oxígeno disminuye, es posible que los nutrientes se acumulen, permaneciendo disponibles para el fitoplancton y estimulando las floraciones, además de generar mortandad de peces y macroinvertebrados. Consecuentemente, el bajo nivel de oxígeno genera preocupación por la salud interna del lago (Chandra et al., 2013).

Otra variable importante a nivel biológico es la temperatura, Basterrechea (1983) reportó temperatura promedio anual de $20{ }^{\circ} \mathrm{C}$ en el lago de Amatitlán, hace 36 años. Las temperaturas reportadas en el presente estudio, fueron de $24{ }^{\circ} \mathrm{C}$ en promedio anual. El aumento de la temperatura de las aguas superficiales provoca intensificación de las floraciones de cianobacterias, ya que, estas especies se adaptan rápidamente a altas temperaturas (Basterrechea et al., 2019; Raven \& Kübler, 2002). Además, las altas temperaturas aumentan la estabilidad de la columna de agua lo que beneficia a las cianobacterias flotantes al reducir la mezcla vertical y, por ende, el equilibrio competitivo entre el fitoplancton (Huisman et al., 2011). Así mismo, los valores de pH alcalinos, de hasta 9.5, obtenidos en el presente estudio favorecieron el desarrollo de las cianobacterias, ya que, estas tienen la capacidad de transformar los iones bicarbonato y carbonato en dióxido de carbono, y de desarrollar biomasas importantes en ambientes naturalmente alcalinos, también poseen una gran capacidad para absorber dióxido de carbono disuelto, aún en concentraciones muy bajas (Basterrechea et al., 2019; Raven \& Kübler, 2002).

Existen otras variables fisicoquímicas determinantes del estado trófico de los cuerpos de agua, e importantes para las poblaciones bióticas, como el NT y PT. En el punto RM se presentó la menor riqueza de fitoplancton (18 géneros) y la mayor abundancia de cianobacterias del género Microcystis (69.08\%; Tabla 4). Esto puede ser debido a que el desarrollo de los procesos eutróficos por el aumento en la concentración de nutrientes está promoviendo la expansión y persistencia de cianobacterias, por su rápida capacidad adaptativa a las condiciones ecológicas cambiantes (Huisman et al., 2011; Komárek et al., 2013; Paerl \& Huisman, 


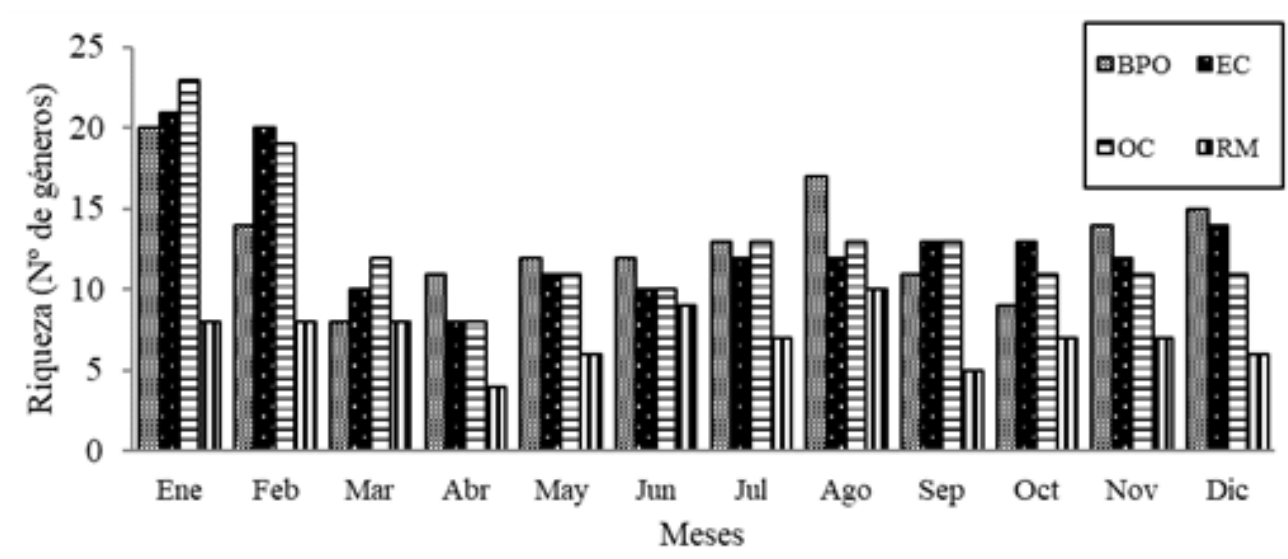

Figura 2. Riqueza expresada en número de géneros por meses y sitios de muestreo. Abreviaturas para puntos de muestreo: Lago de Amatitlán este centro (EC), Lago de Amatitlán oeste centro (OC), bahía Playa de Oro (BPO) y río Michatoya (RM).

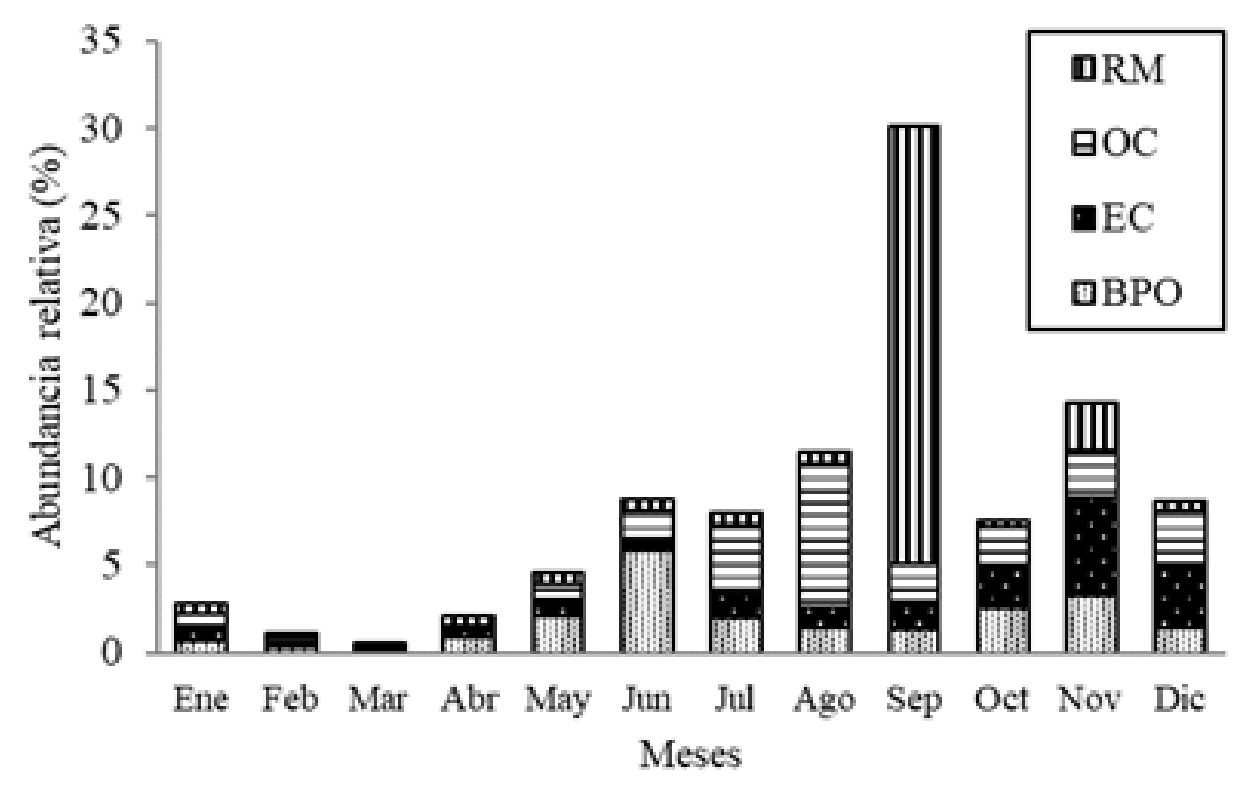

Figura 3. Abundancia relativa de los géneros de fitoplancton encontrado por punto de muestreo y mes del 2017. Abreviaturas para puntos de muestreo: Lago de Amatitlán este centro (EC), Lago de Amatitlán oeste centro (OC), bahía Playa de Oro (BPO) y río Michatoya (RM).

2009). Igualmente, estos procesos de eutrofización tienen por consecuencia la reducción de la biodiversidad del fitoplancton (Fontúrbel, 2005). El IETP (Carlson, 1977) mostró valores entre 63.8 y 88.4 (Tabla 1), lo que determina que el lago de Amatitlán presenta un estado eutrófico a hipereutrófico, probablemente por los vertimientos de aguas residuales industriales y domésticas provenientes de la ciudad de Guatemala (Calderón, 2010).
En los resultados del análisis cuali-cuantitativo de fitoplancton se registraron 34 géneros, mientras que, Díaz (2011) encontró 18 géneros de microalgas en el sector oeste del lago de Amatitlán, y Peckham y Dineen (1953) registraron 38 géneros en el mismo. El sitio BPO presentó la mayor riqueza de microalgas, Díaz (2011) reportó una mejora en la calidad de agua en esta playa, debido a la presencia de vegetación superficial. Entre tanto, EC y OC mostraron una diversidad de fitoplanc- 


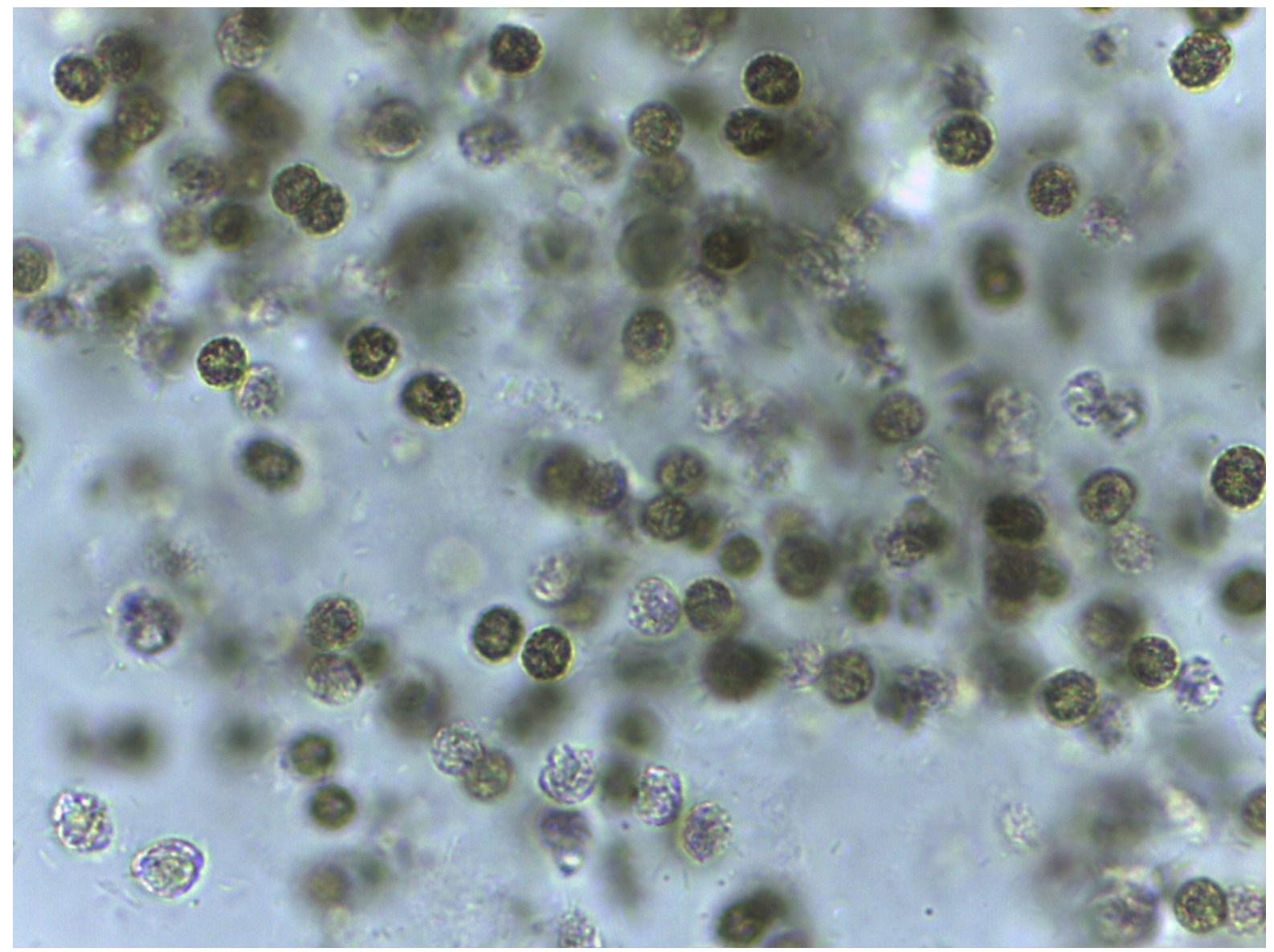

Figura 4. Microcystis sp. Foto a 100X, tomada por: Sara Molina.

ton similar, aunque baja en ambos casos. Es posible que la baja riqueza en todos los puntos de muestreo esté relacionada con el aumento de nutrientes debido a descarga de aguas residuales, así se ha demostrado en otros grandes lagos de Guatemala, como el lago de Atitlán (Chandra et al., 2013).

Así mismo, se reportaron 13 géneros de cianobacterias (Tabla 4), mientras que, Peckham y Dineen (1953) reportaron 11 géneros para el lago de Amatitlán, dentro de los cuales no estaba Mycrocystis, el género dominante en el lago actualmente. En estudios más recientes, Calderón (2010) y Díaz (2011) reportaron a las cianobacterias como grupo dominante del fitoplancton del lago de Amatitlán. Sin embargo, las cianobacterias no son las únicas capaces de sobrevivir a estas condiciones ambientales, las algas verdes (Chlorophyta) también están presentes, aunque en menor proporción ( $0.3 \%$ del total del fitoplancton) De estas, el género Scenedesmus fue el más abundante $(0.026 \%$; Tabla 4$)$, catalogado con capacidad de remoción de nutrientes y metales pesados, además de poseer adaptación a ambientes residuales en condiciones mixotróficas, por lo que ha sido utilizado en biorremediación (Andrade et al., 2009; Monteiro et al., 2009).

Por otro lado, un componente importante del fitoplancton son las diatomeas, algunas especies son consideradas como bioindicadoras de calidad de agua, en este caso se encontraron tres géneros, que se caracterizan por su alta resistencia y desarrollo en variedad de condiciones tróficas (Denys et al., 2003). Dos de ellas (Nitszchia y Aulacoseira) son indicativos de aguas ricas en nutrientes, con alta concentración de solidos totales disueltos y turbidez (Lobo et al., 2015; Taylor et al., 2007). Peckham y Dineen (1953) registraron 11 géneros de diatomeas en el lago de Amatitlán, con dominancia de Melosira sp. (54\% del total del plancton), pero ninguno coincide con los géneros reportados en este estudio. 
Un factor determinante en la composición y estructura del fitoplancton es el periodo hidrológico (Ramírez \& Plata-Díaz, 2008; Rodriguez \& Bicudo, 2001; Tundisi, 1990). En general los primeros meses del año tuvieron mayor riqueza, esta época corresponde a temporada seca, en la cual, es común encontrar una mayor estabilidad de las comunidades fitoplanctónicas, ya que, factores abrasivos como la velocidad del viento y de la corriente, y el caudal de los afluentes disminuyen (Montoya \& Ramírez, 2007; Rodriguez \& Bicudo, 2001; Ramírez \& Plata-Díaz, 2008). A pesar que se marcan dos estaciones, la seca, que va de noviembre a abril y la lluviosa, entre mayo a octubre, los meses de menor riqueza estuvieron entre marzo y abril siendo aún época seca y los meses más cálidos, y durante septiembre y octubre cuando la época lluviosa va llegando a su fin (Gudiel, 2007).

Las variables climáticas como el viento, las precipitaciones y la turbulencia en la columna de agua, que aumentan en período de lluvias, influyen en la disponibilidad de nutrientes y la radiación solar subacuática de un ecosistema, por lo que se observa una variación en la estructura del fitoplancton (Esteves, 1988). Es otras palabras, en época seca, verano o veranillo, se puede encontrar mayor diversidad de microalgas debido a la mayor incidencia de radiación solar, el poco recambio de agua y la acumulación de materia orgánica y nutrientes, que les permite el aumento de metabolismo, y a su vez, de reproducción celular (Tundisi, 1990).

En RM se presenta un aumento desmedido de abundancia relativa de fitoplancton durante septiembre, llegando a un valor cuatro veces más alto que en el resto de sitios, y hasta 200 veces el valor del promedio de los demás meses en ese mismo sitio (Figura 3). Así mismo, se observan aumentos de abundancia en diferentes meses por cada sitio, que se atribuyen mayoritariamente a proliferaciones de Microcystis. Lo que tienen en común los meses donde ocurren dichas proliferaciones son los marcados aumentos de nitrógeno total y fosforo total (datos no mostrados), que aumentan los niveles de eutrofización del lago y son el ambiente propicio para estas cianobacterias (Harke et al., 2012; Chaffin \& Bridgeman, 2014).

La baja diversidad de fitoplancton del lago se comprueba con los índices de diversidad calculados de Shannon-Weaver y Simpson (Tabla 5). RM posee los valores más altos de eutrofización (Tabla 1) y los índices más bajos de diversidad (Tabla 5), aunque no se evidencian diferencias significativas $(p>.5)$ con respecto a los demás sitios de muestreo (Tabla 6). Este comportamiento se ha demostrado en otros estudios donde el estado trófico es responsable de la estructura y composición biótica de un ecosistema acuático (Ferrer et al., 2012; Lugioyo et al., 2007).

El índice de diversidad de Shannon (N1) y la dominancia de Simpson indican a los puntos OC y BPO como los de mayor diversidad dentro del lago (Tabla 5), es posible que la marcada dominancia de Microcystis esté afectando el valor de estos índices. Los valores de estos índices muestran una baja diversidad de fitoplancton en todos los puntos de muestreo del lago de Amatitlán.

Por su parte, la Equitabilidad de Hill se vio afectada en RM, ya que, es un índice susceptible a especies con dominancia significativa, modificando los valores, y pudiendo generar una interpretación incorrecta (Baev \& Penev, 1995). Mientras que, el índice de Pielou mostró a RM como el sitio de menor uniformidad (Tabla 5), en concordancia con los otros índices de diversidad.

Además de la baja riqueza, se observó que el mayor responsable de las floraciones es el género Microcystis ( $38.40 \%$ de abundancia relativa). Esta cianobacteria posee mecanismos de adaptación que le permiten dominar en diversos ambientes y condiciones tróficas, tales como: regulación de flotabilidad que le permite moverse verticalmente por la columna de agua en busca de las mejores condiciones; mecanismos celulares para acceder y almacenar nitrógeno y fósforo intracelular; producción de microcistinas, que provocan la muerte de otros microorganismos y así evitan la competencia por nutrientes, luz y espacio (Harke et al., 2016). De igual forma, se reportó la presencia de Lyngbya $(0.30 \%$ de abundancia relativa), otro género productor de cianotoxinas, que junto a Microcystis son las responsables de numerosos casos de intoxicación en el mundo (Zacolar \& Forastier, 2005).

La otra cianobacteria dominante en el lago fue Pseudanabaena (33.60\% de abundancia relativa), de este género se tiene menos información disponible. Aunque no suele causar grandes floraciones, se ha reportado como dominante en algunos lagos eutróficos de Latinoamérica (Olvera-Ramírez et al., 2010). Pseudanabaena no posee heterocistos para fijación de nitrógeno, pero si presenta vesículas de gas que le permiten moverse por la columna de agua en busca de luz y nutrientes (Damerval et al., 1991). Además, tiene la capacidad de producir cianotoxinas, y otros metabolitos altamente tóxicos para la biota acuática, que puede llegar a modificar la diversidad y dinámica de la cadena trófica (Olvera-Ramírez et al., 2010; Oudra et al., 2002). 
Tabla 4

Composición taxonómica y abundancia relativa del fitoplancton colectado en el lago de Amatitlán, por sitios de muestreo. Se agruparon los datos de meses y profundidades

\begin{tabular}{|c|c|c|c|c|c|c|}
\hline \multicolumn{7}{|c|}{ Abundancia relativa $(\%)$} \\
\hline Familia & Géneros & $\mathrm{BPO}$ & $\mathrm{EC}$ & $\mathrm{OC}$ & $\mathrm{RM}$ & Total \\
\hline \multirow{3}{*}{ Aphanizomenonaces } & Aphanizomenon & 0.283 & 0.363 & 0.053 & 0.000 & 0.146 \\
\hline & & & & & & \\
\hline & Dolichospermum & 0.149 & 1.646 & 0.081 & 0.028 & 0.387 \\
\hline Aphanothecaceae & Aphanothece & 0.055 & 0.078 & 0.012 & 0.000 & 0.030 \\
\hline Aulacoseiraceae & Aulacoseira & 0.551 & 0.199 & 0.399 & 0.009 & 0.259 \\
\hline Bacillariaceae & Nitzschia & 0.203 & 0.030 & 0.101 & 0.049 & 0.091 \\
\hline Characiaceae & Characium & 0.007 & 0.000 & 0.002 & 0.000 & 0.002 \\
\hline \multirow[t]{2}{*}{ Chlorangiellaceae } & Stylosphaeridium & 0.003 & 0.016 & 0.010 & 0.000 & 0.006 \\
\hline & Actinastrum & 0.134 & 0.024 & 0.182 & 0.000 & 0.078 \\
\hline \multirow[t]{2}{*}{ Chlorellaceae } & Dictyosphaerium & 0.082 & 0.000 & 0.000 & 0.000 & 0.018 \\
\hline & Micractinium & 0.096 & 0.036 & 0.000 & 0.000 & 0.028 \\
\hline Closteraceae & Closterium & 0.006 & 0.011 & 0.000 & 0.009 & 0.006 \\
\hline Coelosphaeriaceae & Snowella & 0.000 & 0.017 & 0.002 & 0.000 & 0.004 \\
\hline Desmidiaceae & Cosmarium & 0.002 & 0.000 & 0.008 & 0.000 & 0.002 \\
\hline Dinobryaceae & Chrysococcus & 0.008 & 0.005 & 0.007 & 0.000 & 0.005 \\
\hline Elakatotrichaceae & Elakatothrix & 0.113 & 0.002 & 0.008 & 0.000 & 0.027 \\
\hline Hydrodictyaceae & Tetraedron & 0.023 & 0.028 & 0.051 & 0.002 & 0.024 \\
\hline \multirow{2}{*}{ Merismopediaceae } & Aphanocapsa & 3.522 & 6.061 & 5.411 & 2.998 & 4.312 \\
\hline & Merismopedia & 14.575 & 12.257 & 11.793 & 6.690 & 10.747 \\
\hline Microcoleaceae & Planktothrix & 0.094 & 0.043 & 0.164 & 0.005 & 0.071 \\
\hline Microcystaceae & Microcystis & 31.816 & 10.992 & 31.382 & 69.089 & 38.405 \\
\hline
\end{tabular}


Tabla 4 (Continuación)

\begin{tabular}{|c|c|c|c|c|c|c|}
\hline \multicolumn{7}{|c|}{ Abundancia relativa (\%) } \\
\hline Familia & Géneros & $\mathrm{BPO}$ & $\mathrm{EC}$ & $\mathrm{OC}$ & $\mathrm{RM}$ & Total \\
\hline \multirow{2}{*}{ Oocystaceae } & Chodatella & 0.000 & 0.001 & 0.000 & 0.000 & 0.000 \\
\hline & Oocystis & 0.004 & 0.001 & 0.012 & 0.000 & 0.004 \\
\hline Oscillatoriaceae & Lyngbya & 0.000 & 0.000 & 0.117 & 0.002 & 0.030 \\
\hline Pseudanabaenaceae & Pseudanabaena & 39.506 & 59.129 & 35.163 & 13.882 & 34.993 \\
\hline Pyrenomonadaceae & Rhodomonas & 0.082 & 0.092 & 0.073 & 0.027 & 0.063 \\
\hline Scenedesmaceae & Scenedesmus & 0.093 & 0.013 & 0.015 & 0.000 & 0.026 \\
\hline \multirow{2}{*}{ Selenastraceae } & Kirchneriella & 0.214 & 0.063 & 0.118 & 0.036 & 0.100 \\
\hline & Monoraphidium & 0.007 & 0.012 & 0.000 & 0.001 & 0.004 \\
\hline Spirulinaceae & Spirulina & 0.369 & 0.869 & 2.100 & 0.238 & 0.850 \\
\hline Stephanodiscaceae & Cyclotella & 0.056 & 0.029 & 0.022 & 0.019 & 0.030 \\
\hline \multirow{3}{*}{ Synechococcaceae } & Cyanodictyon & 3.975 & 4.834 & 6.753 & 3.302 & 4.601 \\
\hline & & & & & & \\
\hline & Synechocystis & 3.949 & 3.126 & 5.948 & 3.616 & 4.166 \\
\hline Trebouxiophyceae & Crucigenia & 0.007 & 0.024 & 0.017 & 0.000 & 0.010 \\
\hline Volvocaceae & Pandorina & 0.016 & 0.000 & 0.000 & 0.000 & 0.004 \\
\hline \multicolumn{2}{|c|}{ Total } & 100 & 100 & 100 & 100 & 100 \\
\hline
\end{tabular}

Nota. Abreviaturas para puntos de muestreo: Lago de Amatitlán este centro (EC), Lago de Amatitlán oeste centro (OC), bahía Playa de Oro (BPO) y río Michatoya (RM).

Para evaluar el efecto de la calidad de agua sobre la biodiversidad del fitoplancton se utilizó un análisis de redundancia (RDA). Los tres únicos géneros de diatomeas encontrados en el estudio fueron Nitzschia (Nit), Aulacoseira (Aul) y Cyclotella (Cyc) y mostraron cercanía entre sí en el gráfico de ordenación RDA, asociados positivamente con el aumento de $\mathrm{pH}$ y tensoactivos (Figura 6). La diatomea más abundante fue Nitzschia, la cual es considerada como indicadora de una baja calidad de agua por su resistencia a la eutrofización (Lobo et al., 2015). Las diatomeas son un grupo muy diverso dentro de las microalgas, su presencia es común en ambientes conservados, por ello, encontrar solo tres géneros, que son altamente tolerantes a cambios ambientales como el aumento de concentración de nutrientes, es un indicador del impacto antrópico que se está generando (Bicudo et al., 2016; Reynolds, 1984; Vera-Parra et al., 2011). 
Tabla 5

Índices de diversidad y números de Hill (1973), calculados con los datos de abundancia absoluta (células/ml) de los géneros de fitoplancton encontrados en el lago de Amatitlán, durante 2017

\begin{tabular}{|c|c|c|c|c|c|}
\hline \multirow{2}{*}{ Sitios de muestreo } & Riqueza & Shannon & Simpson & \multirow{2}{*}{$\begin{array}{c}\text { Equitabilidad } \\
\text { de Hill }\end{array}$} & \multirow{2}{*}{ Pielou } \\
\hline & $(\mathrm{N} 0)$ & $(\mathrm{N} 1)$ & (N2) & & \\
\hline $\mathrm{BPO}$ & 31 & 4.909 & 1.381 & 0.281 & 0.463 \\
\hline $\mathrm{EC}$ & 29 & 4.096 & 1.626 & 0.397 & 0.419 \\
\hline $\mathrm{OC}$ & 29 & 5.155 & 1.346 & 0.261 & 0.487 \\
\hline RM & 18 & 3.010 & 1.969 & 0.654 & 0.381 \\
\hline
\end{tabular}

Nota. Abreviaturas para puntos de muestreo: Lago de Amatitlán este centro (EC), Lago de Amatitlán oeste centro (OC), bahía Playa de Oro (BPO) y río Michatoya (RM).

Tabla 6

Pruebas de significancia estadística de la composición y diversidad del fitoplancton del Lago de Amatitlán

\begin{tabular}{lll}
\hline \multicolumn{1}{c}{ Índice } & Valor-P & Prueba \\
\hline Riqueza & $8.85 \mathrm{E}-05$ & ANOVA \\
Riqueza sin RM & 0.9819 & ANOVA \\
Abundancia & 0.2857 & Kruskal-Wallis \\
Shannon-W & 0.3251 & ANOVA \\
Simpson & 0.5674 & ANOVA \\
Pielou & 0.2585 & ANOVA \\
\hline
\end{tabular}

Para el caso del análisis RDA de cianobacterias, los géneros agrupados entre sí (Pseudanabaena, Merismopedia, Aphanocapsa y Synechocystis) tienen en común ser productores de cianotoxinas. Por otra parte, Microcystis, muestra relación directa con el aumento de nutrientes como nitrógeno total, fósforo total y demanda química de oxígeno (Figura 6), confirmando su adaptación a condiciones eutróficas. De igual manera, la profundidad se observa como una variable explica- tiva ya que en la parte superficial del lago se encuentra la mayor concentración de Microcystis, mientras que, en zonas más profundas se encuentran la mayor abundancia de las demás cianobacterias. Probablemente, la capacidad de migración vertical de Microcystis, hacia la superficie en busca de luz o hacia zonas más profundas para obtener nutrientes, le permite una ventaja adaptativa sobre las demás cianobacterias (Harke et al., 2016).

En conclusión, los aumentos de coliformes fecales, DQO, NT y PT, potencian las proliferaciones de cianobacterias en el lago de Amatitlán, al igual que lo reportado por Paerl y Huisman (2009) en otros sistemas. Los altos niveles de eutrofización y el consecuente aumento de cianobacterias tóxicas están generando problemáticas ambientales en este lago, al igual que en ecosistemas acuáticos de todo el mundo (Harke et al., 2012), por lo que es necesario conocer las causas y consecuencias del problema y establecer posibles soluciones. Como indicadores biológicos de la contaminación del lago de Amatitlán se encontraron la dominancia de Microcystis, la baja diversidad de diatomeas, la presencia de fitoplancton adaptado a procesos de eutrofización, como Nitzschia y Scenedesmus, y la alta concentración de coliformes fecales.

En este estudio encontramos la presencia de Scenedesmus. Algunas especies de este género poseen capacidad de remover nitratos, fosfatos, materia orgánica (Chacón et al., 2004; Parra \& Villanueva, 2012) y metales pesados (Monteiro et al., 2009), se 


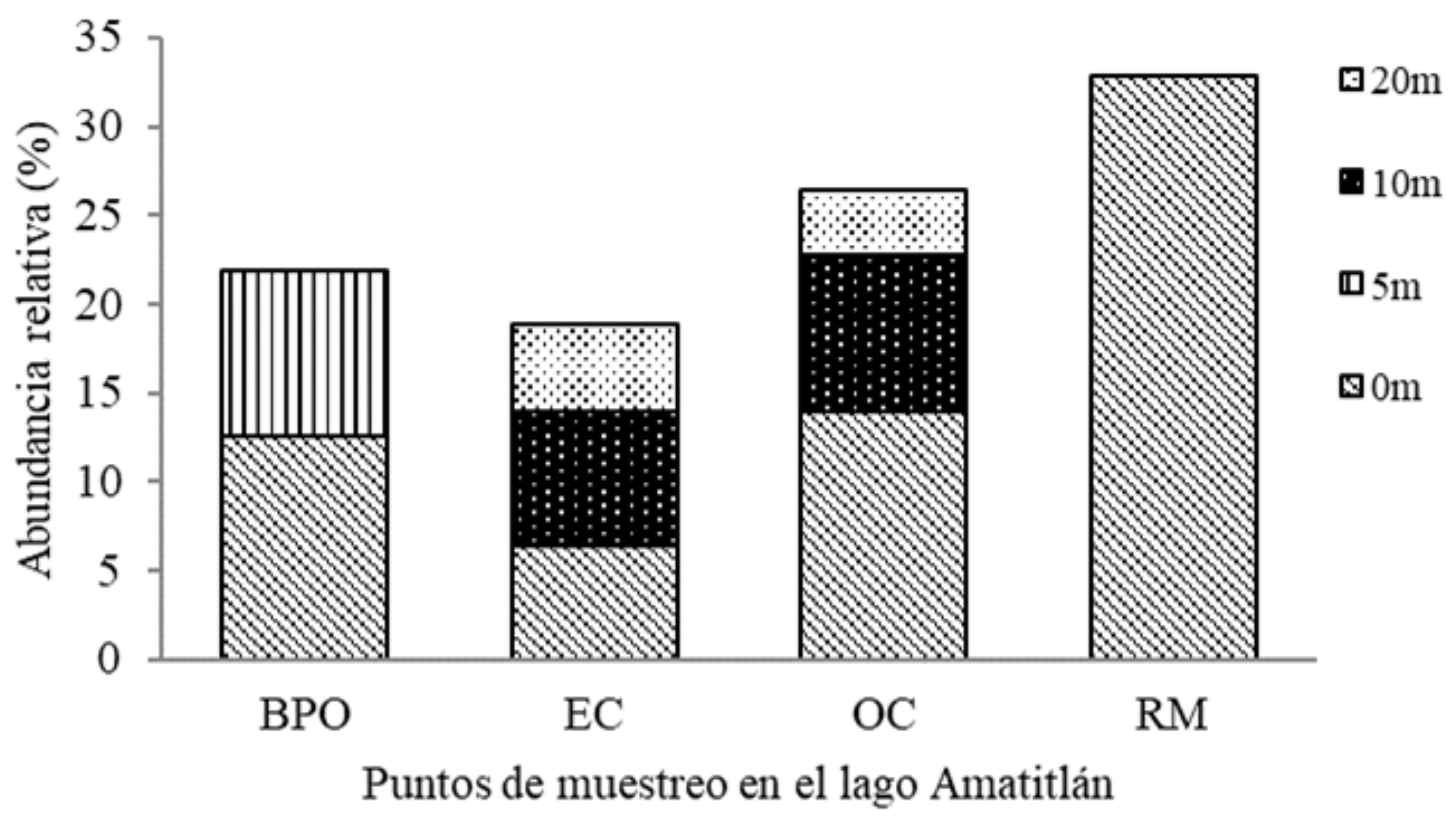

Figura 5. Abundancia relativa de los géneros de fitoplancton a diferentes profundidades, por sitio de muestreo.
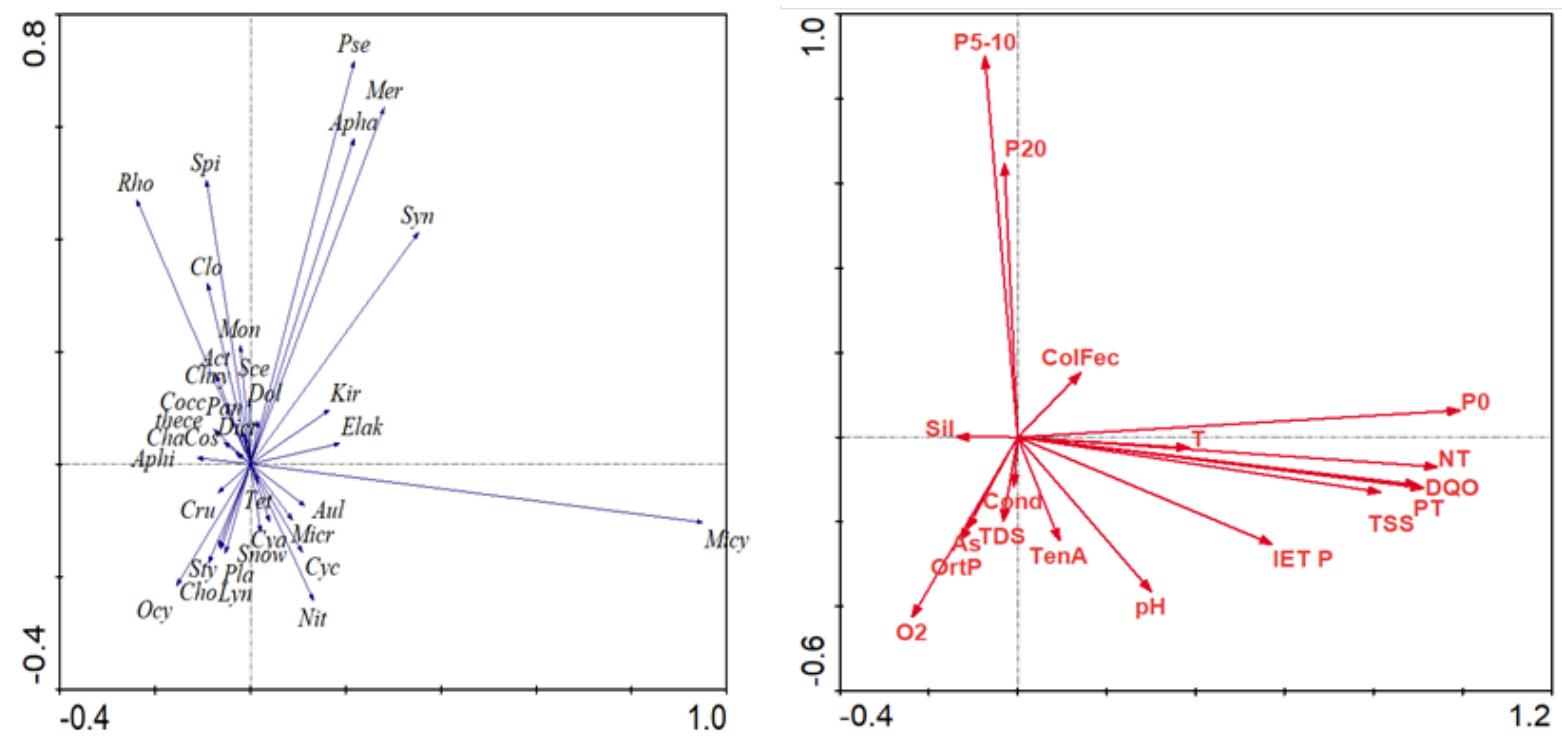

Figura 6. Análisis de redundancia (RDA) de los géneros de microalgas con respecto a las variables ambientales reportadas en el lago de Amatitlán durante el 2017. Abreviaturas de parámetros: As: arsénico; Clor: clorofila a; ColFec: coliformes fecales; Cond: conductividad; DQO: demanda química de oxigeno; IETP: índice de estado trófico; NT: nitrógeno total; $\mathrm{O}_{2}$ : oxígeno disuelto; OrtP: ortofosfatos; PT: fosforo total; Sil: Silicatos; SST: Solidos totales suspendidos; T: temperatura; TDS: solidos totales disueltos; TenA: tensoactivos. Abreviaturas de géneros de fitoplancton: Actinastrum: Act; Cyanodictyon: Cya; Nitzschia: Nit; Aphanizomenon: Aphi; Cyclotella: Cyc; Oocystis: Ocy; Aphanocapsa: Apha; Dictyosphaerium: Dict; Pandorina: Pan; Aphanothece: thece; Dolichospermum: Dol; Planktothrix: Pla; Aulacoseira: Aul; Elakatothrix: Elak; Pseudanabaena: Pse; Characium: Cha; Golenkinia: Gol; Rhodomonas: Rho; Chodatella: Cho; Kirchneriella: Kir; Scenedesmus: Sce; Chrysococcus: Chry; Lyngbya: Lyn; Snowella: Snow; Closterium: Clo; Merismopedia: Mer; Spirulina: Spi; Coccoid cell: Cocc; Micractinium: Micr; Stylosphaeridium: Sty; Cosmarium: Cos; Microcystis: Micy; Synechocystis: Syn; Crucigenia: Cru; Monoraphidium: Mon; Tetraedron: Tet. 
recomienda continuar con la búsqueda de otras especies con potencial para biorremediación en el lago de Amatitlán, y realizar ensayos que comprueben dicha capacidad. Igualmente, se debe tener más control sobre las aguas residuales que llegan al lago y así mitigar la eutrofización que se está presentando actualmente.

\section{Agradecimientos}

A Amed Juárez, Director de Autoridad para el Manejo Sustentable de la Cuenca y del Lago de Amatitlán (AMSA), a Manuel Cano, Jefe de la División de Control, Calidad Ambiental y Manejo de Lagos y a todos sus colaboradores en el Laboratorio y en la Estación Acuática en especial a Julio R. Juárez, Elena Reyes, Alicia Toledo y Marlon Alonzo. A Sara Molina profesora de la Universidad Mariano Gálvez.

\section{Referencias}

Andrade, C., Vera, A., Cárdenas, C., \& Morales, E. (2009). Biomass production of microalga Scenedesmus sp. with wastewater from fishery. Revista Técnica de la Facultad de Ingeniería Universidad del Zulia, 32(2), 126-134.

Baev, P. V., \& Penev, L. D. (1995). BIODIV: Program for calculating biological diversity parameters, similarity, niche overlap, and cluster analysis. Versión 5.1. Pensoft, Sofia, Bulgaria.

Basterrechea, M. (September 1983). Modelo para Estimar la calidad del Agua en los Lagos: El Lago de Amatitlán. 187-202. Proceedings of the Tegucigalpa Hydromath Symposium.(IAHS Publ. No. 152). Recuperado de http://hydrologie.org/ redbooks/a152/iahs_152_0187.pdf

Basterrechea, M., Dix, M., van Tuylen, S., Méndez, Á., Díaz, L., Mayorga, P., \& Gil, N. (2019). Calidad del agua en Guatemala. En Calidad del Agua en las Américas riesgos y oportunidades (pp. 375392). IANAS. Recuperado de https://www.ianas. org/images/books/wb09.pdf

Bellinger, E. G., \& Sigee, D. C. (2015). Freshwater Algae: Identification, enumeration and use as bioindicators (2nd. ed.). Manchester, UK: WileyBlackwell.
Bicudo, D. C., Tremarin, P. I., Almeida, P. D., Zorzal-Almeida, S., Wengrat, S., Faustino, S. B., ... Morales, E. A. (2016). Ecology and distribution of Aulacoseira species (Bacillariophyta) in tropical reservoirs from Brazil. Diatom Research, 31(3), 199-215. https:// doi.org/10.1080/0269249X.2016.1227376

Calderón, I. M. (2010). Green movement against Green water: Using green infrastructure strategies in the lake Amatitlán watershed to promote environmental education in Guatemala Honors (Tesis de doctorado). Cornell University, Ithaca, New York.

Carlson, R. E. (1977). A trophic state index for lakes 1. Limnology and Oceanography, 22(2), 361-369. https://doi.org/10.431/lo.1977.22.2.0361

Chacón, C., Andradel, C., Cárdenas, C., Araujo, I. \& Morales, E. (2004). Uso de Chlorella sp. y Scenedesmus sp. en la remoción de nitrógeno, fósforo y DQO de aguas residuales urbanas de Maracaibo, venezuela. Boletín del Centro de Investigaciones Biológicas, 38(2), 94-108.

Chaffin, J. D., \& Bridgeman, T. B. (2014). Organic and inorganic nitrogen utilization by nitrogen stressed cyanobacteria during bloom conditions. Journal of Applied Phycology, 26, 299-309. https://doi. org/10.1007/s10811-013-0118-0

Chandra, S., Dix, M., Rejmánková, E., Mosquera, V., Girón, N., \& Heyvaert, A. (2013). El estado ecológico actual del Lago Atitlán y el impacto de la entrada de aguas residuales: Recomendación para exportación de las aguas residuales de la cuenca para restaurar el lago. Recuperado de http:// agualimpiaya.org/wp-content/uploads/2019/01/ Reporte-Cientifico-2013x-1.pdf

Clesceri, L. S., Greenberg, A. E., Eaton, A. D., Rice, E. W., \& Franson, M. A. H. (2005). Standard methods for the examination of water and wastewater (No. 628.161 S7/2005). Washington, DC: American Public Health Association.

Cronberg, G., \& Annadotter, H. (2006). Manual on aquatic cyanobacteria: A photo guide and a sypnosis of their toxicology. Copenhagen: International Society for the Study of Harmful Algae-United Educational, Scientific and Cultural Organization. 
Damerval, T., Castets, A. M., Houmard, J., \& De Marsac, N. T. (1991). Gas vesicle synthesis in the cyanobacterium Pseudanabaena sp.: Occurrence of a single photoregulated gene. Molecular Microbiology, 5(3), 657-664. https://doi. org/10.1111/j.1365.2958.199.tb00737.x

Denys, L., Muylaert, K., Krammer, K., Joosten, T., Reid, M., \& Rioual, P. (2003). Aulacoseira subborealis stat. nov. (Bacillariophyceae): A common but neglected plankton diatom. Nova Hedwigia, 77(3/4), 407-427. https://doi.org/10.1127/00295035/2003/0077-0407

Díaz, J. M. (2011). Evaluación de pre-factibilidad técnico-económica de las potencialidades energéticas de las microalgas que contaminan el lago Amatitlán para la obtención de biodiesel (Fodecyt No. 049-2009). Guatemala: Consejo Nacional de Ciencia y Tecnología y Universidad Galileo.

Esteves, F. (1988). Fundamentos de Limnología. Río de Janeiro, Brasil: Interciencia.

Falconer, I. R. (2005). Is there a human health hazard from microcystins in the drinking water supply? Acta Hydrochimica et Hydrobiologica, 33(1), 64-71. https://doi.org/10.1002/aheh.200300551

Ferrer, N. C., Cony, N. L., Fornerón, C. F., \& Piccolo, M. C. (2012). Caracterización del fitoplancton y estado trófico de la laguna Sauce Grande (Provincia de Buenos Aires, Argentina) en el otoño de 2010. Biología Acuática. 27, 129-141

Fontúrbel, F. (2005). Indicadores fisicoquímicos y biológicos del proceso de eutrofización del Lago Titikaka (Bolivia). Ecología Aplicada, 4(1-2), 135-141.

Gómez, L. M. (2007). Microalgas: Aspectos ecológicos y biotecnológicos. Revista Cubana de Química, 19(2), 3-20.

Gómez, L., Larduet, Y., \& Abrahantes, N. (2001). Contaminación y biodiversidad en ecosistemas acuáticos. El fitoplancton de la bahía de Santiago de Cuba. Revista de Investigaciones Marinas, 22(3), 191-197.

Gudiel, D. (2007). Evaluación del efecto de la aireación artificial para mejorar la calidad del agua en el lago de Amatitlán. (Tesis de pregrado). Facultad de Ciencias Químicas y Farmacia. Universidad de San Carlos de Guatemala. Guatemala
Hammer, Ø., Harper, D. A., \& Ryan, P. D. (2001). PAST: Paleontological statistics software package for education and data analysis. Palaeontologia Electronica, 4(1), 9.

Harke, M. J., Berry, D. L., Ammerman, J. W., \& Gobler, C. J. (2012). Molecular response of the bloomforming cyanobacterium, Microcystis aeruginosa, to phosphorus limitation. Microbiology Ecology, 63(1), 188-198. https://doi.org/10.1007/s00248011-9894-8

Harke, M. J., Steffen, M. M., Gobler, C. J., Otten, T. G., Wilhelm, S. W., Wood, S. A., \& Paerl, H. W. (2016). A review of the global ecology, genomics, and biogeography of the toxic cyanobacterium, Microcystis spp. Harmful Algae, 54, 4-20. https:// doi.org/10.1017/j.hal.2015.12.007

Henriques, M., Silva, A., \& Rocha, J. M. (2007). Extraction and quantification of pigments from a marine microalga: A simple and reproducible method. Communicating Current Research and Educational Topics and Trends in Applied Microbiology Formatex, 2, 586-593.

Hill, M. O. (1973). Diversity and evenness: A unifying notation and its consequences. Ecology, 54, 427-432. http://doi.org/10.1590/S010084042001000300001

Huisman, J., Paerl, H. W., Joehnk, K., Van de Waal, D. B., Visser, P. M., \& Verspagen, J. M. (2011). Harmful cyanobacteria: Favored by global warming but suppressed by rising $\mathrm{CO}_{2}$ ? 8th APGC Symposium, Groningen, the Netherlands.

Jost, L. (2006). Entropy and diversity. Oikos, 113(2), 363-375. https://doi.org/10.1111/j.2006.00301299.14714.x

Komárek, J., Zapomělová, E., Šmarda, J., Kopecký, J., Rejmánková, E., Woodhouse, J., ... \& Komarkova, J. (2013). Polyphasic evaluation of Limnoraphis robusta, a water-bloom forming cyanobacterium from Lake Atitlán, Guatemala, with a description of Limnoraphis gen. nov. Fottea, 13(1), 39-52. https://doi.org/10.5507/fot.2013.004

Komárková, J., Montoya, H., \& Komárek, J. (2016). Cyanobacterial water bloom of Limnoraphis robusta in the Lago Mayor of Lake Titicaca. Can it develop? Hydrobiologia, 764(1), 249-258. https://doi.org/10.1007/s10750-015-2298-x 
Leps, J., \& Smilauer, P. (2003). Multivariate analysis of ecological data using CANOCO. New York: Cambridge University Press.

Lobo, E. A., Schuch, M., Heinrich, C. G., da Costa, A. B., Düpont, A., Wetzel, C. E., \& Ector, L. (2015). Development of the Trophic Water Quality Index (TWQI) for subtropical temperate Brazilian lotic systems. Environmental Monitoring and Assessment, 187(6), 354. https://doi.org/10.1007/ s10661-015-4586-3

Lucena, E. (2008). Aspectos sanitarios de las cianotoxinas. Higiene y Sanidad Ambiental, 8, 291-302.

Lugioyo, G. M., Miravet M. E., Pérez R., Álvarez C., \& Espinosa G. (2007). Evaluación del estado trófico de las aguas oceánicas adyacentes al sur de Cuba a partir de indicadores microbiológicos y fitoplanctónicos. Revista de Investigaciones Marinas, 28(3), 201-207.

Michels, A., Lloyd, M., Woeller, R., Dionne, J. F., Grande, L., Sorto, M., \& Basterrechea, M. (2014). Sedimentation and life expectancy of lake Amatitlán, Guatemala: increased vulnerability under future climate change. En International Perspectives on Climate Change (pp. 47-60). Cham: Springer,

Monteiro, C. M., Castro, P. M. L., \& Malcata, F. X. (2009). Use of the microalga Scenedesmus obliquus to remove cadmium cations from aqueous solutions. World Journal of Microbiology and Biotechnology, 25(9), 1573-1578. https://doi. org/10.1007/s11274-009-0046-y

Montoya, Y., \& Ramirez, J. (2007). Variación estructural de la comunidad perifitica colonizadora de sustratos artificiales en la zona de ritral del río Medellin, Colombia. Revista de Biología Tropical, 55(2), 585-593.

Müller, R., \& Weidemann. (1955). Die Bestimmung des Nitrats in Wasser. Jahrbuchfur Wasserchemie und Wasserreinigungs-technik. Verlag Chemie, Reinbek, 12, 247-271

Murillo, M. O., Villanueva, R. A., Morales, R. H., \& Heredia, J. D. S. (2010). Evolución trófica de un lago tropical hiposalino en México con base al fitoplancton. Biológicas Revista de la DES Ciencias Biológico Agropecuarias, 12(2), 75-81. nitrogen-stressed cyanobacteria during bloom conditions. Journal of Applied
Olvera-Ramírez, R., Centeno-Ramos, C., \& MartínezJerónimo, F. (2010). Efectos tóxicos de Pseudanabaena tenuis (Cyanobacteria) en los cladóceros Daphnia magna y Ceriodaphnia dubia. Hidrobiológica, 20(3), 203-212.

Oudra, B., Loudiki, M., Vasconcelos, V., Sabour, B., Sbiyyaa, B., Oufdou, K., \& Mezrioui, N. (2002). Detection and quantification of microcystins from cyanobacteria strains isolated from reservoirs and ponds in Morocco. Environmental Toxicology: An International Journal, 17(1), 32-39. https:// doi.org/10.1002/tox.10029

Paerl, H. W., \& Huisman, J. (2009). Climate change: A catalyst for global expansion of harmful cyanobacterial blooms. Environmental Microbiology Reports, 1(1), 27-37. https://doi. org/10.1111/j.1758-2229.2008.00004.x

Parra, A. L. R., \& Villanueva, R. O. C. (2012). Bioremediacion de aguas con fosfatos $\mathrm{y}$ nitratos utilizando Scenedesmus incrasssatulus inmovilizado. Bistua: Revista de la Facultad de Ciencias Básicas, 10(1), 71-79. https://doi. org/10.24054/01204211.v1.n1.2012.50

Peckham, R. S., \& Dineen, C. (1953). Summer Plankton of Lake Amatitlan, Guatemala. The American Midland Naturalist, 50(2), 377-381. https://doi. org/10.2307/2422095

Plataroti, M. C., \& Patiño, P. (2010). Caracterización de la calidad del agua de una sección del Río Luján: Efectos sobre el fitoplancton (Tesis de licenciatura). Universidad de Buenos Aires, Buenos Aires, Argentina.

Prosperi, C., Rodríguez, C., Pierotto, M., Mancini, M., Daga, C., \& Gonella, M. (2005). Evaluación de la contaminación y eutrofización de aguas superficiales de la provincia de Córdoba. Temas de Ciencia y Tecnología, 2(7), 4.

Quiroz, H., Mora, L. M., Molina, I., \& García, J. (2004). Variación de los organismos fitoplanctónicos y la calidad del agua en el lago de Chapala, Jalisco, México. Acta Universitaria, 14(1), 47-58.

Ramírez, A. M., \& Plata-Díaz, Y. (2008). Diatomeas perifíticas en diferentes tramos de dos sistemas lóticos de alta montaña (Páramo de Santurbán, Norte de Santander, Colombia) y su relación con las variables ambientales. Acta Biológica Colombiana, 13(1), 199-216. 
Raven, J. A., \& Kübler, J. E. (2002). New light on the scaling of metabolic rate with the size of algae. Journal of Phycology, 38(1), 11-16. https:// doi.org/10.1046/j.1529-8817.2002.01125.x

Reynolds, C. S. (1984). The ecology of freshwater phytoplankton. New York, USA: Cambridge University Press.

Rodrigues, L., Bicudo, D. C., \& Moschini-Carlos, V. (2003). O papel do perifíton em áreas alagáveis e nos diagnósticos ambientais. En S. M. Thomza \& L. M. Bini (Eds.), Ecologia e manejo de macrófitas aquáticas (pp. 211-230). Maringá: Eduem.

Rodriguez, L., \& Bicudo, D. de C. (2001). Similarity among periphyton algal communities in a lenticlotic gradient of the upper Paraná river floodplain, Brazil. Brazilian Journal of Botany, 24(3), 235248.

Romero-Oliva, C. S., Contardo-Jara, V., Block, T., \& Pflugmacher, S. (2014). Accumulation of microcystin congeners in different aquatic plants and crops-A case study from lake Amatitlán, Guatemala. Ecotoxicology and Environmental Safety, 102(1), 121-128. https://doi.org/10.1016/j. ecoenv.2014.01.031
Taylor, J. C., Harding, W. R., \& Archibald, C. G. M. (2007). An illustrated guide to some common diatom species from South Africa. Pretoria: Water Research Commission.

Tundisi, J. G. (1990). Distribuição espacial, seqüência temporal e ciclo sazonal do fitoplâncton em represas: Fatores limitantes e controladores. Revista Brasileira de Biología, 50(4), 937-955.

Vera-Parra, N. F., Marciales-Caro, L. J., OteroPaternina, A. M., Cruz-Casallas, P. E., \& Velasco-Santamaría, Y. M. (2011). Impacto del agua asociada a la producción de una explotación petrolera sobre la comunidad fitoperifítica del rio Acacias (Meta, Colombia) durante la temporada de lluvias. Orinoquia, 15(1), 31-40.

Zacolar, Y. \& Forastier, M. E. (2005). Biodiversidad de Cyanophyceae (Cyanobacteria) y especies toxigénicas del litoral fluvial argentino. Miscelánea, 14, 213-228. 